Document downloaded from:

http://hdl.handle.net/10251/56197

This paper must be cited as:

Magreñán Ruiz, ȦA.; Cordero Barbero, A.; Gutiérrez Jiménez, JM.; Torregrosa Sánchez, JR. (2014). Real qualitative behavior of a fourth-order family of iterative methods by using the convergence plane. Mathematics and Computers in Simulation. 105:49-61.

doi:10.1016/j.matcom.2014.04.006.

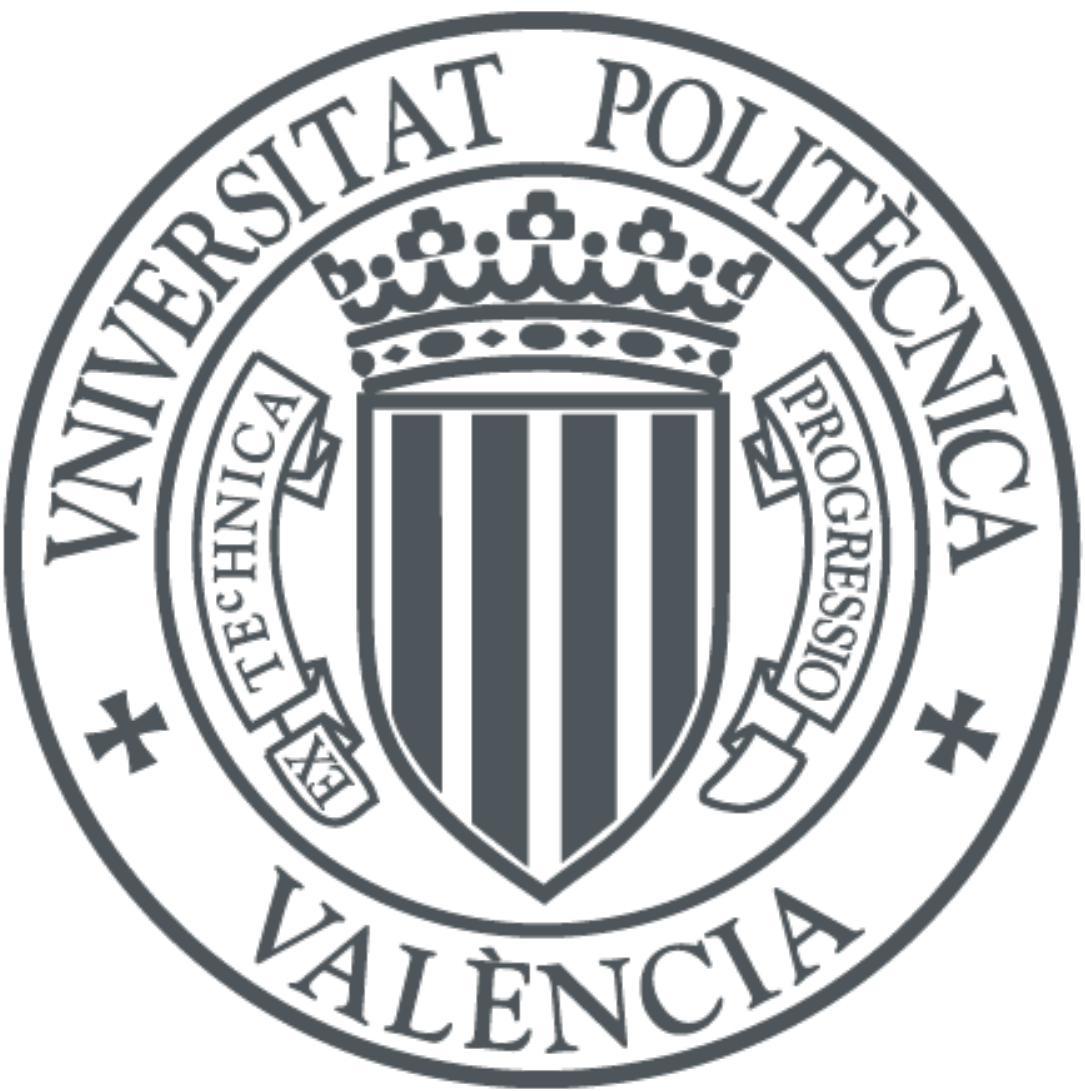

The final publication is available at

http://dx.doi.org/10.1016/j.matcom.2014.04.006

Copyright Elsevier

Additional Information 


\title{
Real qualitative behavior of a fourth-order family of iterative methods by using the convergence plane
}

\author{
Alberto Magreñán ${ }^{\mathrm{a}, *}$, Alicia Cordero ${ }^{\mathrm{b}}$, José M. Gutiérrez ${ }^{\mathrm{a}}$, Juan R. Torregrosa ${ }^{\mathrm{b}}$ \\ ${ }^{a}$ Departamento de Matemáticas y Computación \\ Universidad de La Rioja \\ 26002 Logroño, La Rioja, Spain \\ ${ }^{b}$ Instituto Universitario de Matemática Multidisciplinar \\ Universitat Politècnica de València \\ Camino de Vera s/n, 46022 València, Spain
}

\begin{abstract}
The real dynamics of a family of fourth-order iterative methods is studied when it is applied on quadratic polynomials. A Scaling Theorem is obtained and the conjugacy classes are analyzed. The convergence plane is used to obtain the same kind of information as from the parameter space, and even more, in complex dynamics.
\end{abstract}

Keywords: Real dynamics, nonlinear problems, Convergence Plane, iterative methods, basins of attraction, stability

\section{Introduction}

The application of iterative methods for solving nonlinear problems $f(x)=0$, with $f: \mathbb{R} \rightarrow \mathbb{R}$, gives rise to rational functions whose dynamics are not well-known. The simplest model is obtained when $f(x)$ is a quadratic polynomial and the iterative algorithm is Newton's scheme. This case has been widely studied under the view of complex dynamics (see, for instance $[8,13])$. The study of the dynamics of Newton's method has been extended to other point-to-point iterative schemes (see for example [9, 12, 16, 22]) and to multipoint iterative methods (see for example [1, 2, 10, 11, 21, 23]), for solving nonlinear equations. Nevertheless, the real dynamical analysis is not profusely studied, although some references can be found in the literature (see, for example [6], [14], [3], [4], [17] and [18]).

From the numerical point of view, the dynamical properties of the rational function associated with an iterative method give us important information about its stability and reliability. In most of mentioned papers, interesting dynamical planes, including periodical behavior and other anomalies, have been obtained. We are interested in the analysis of the role of the parameter in the stability of the family of iterative methods, which would allow us to select a particular one with good numerical properties.

In this work, the family under study is the class of three-step fourth-order iterative methods for solving nonlinear systems, introduced by the authors in [5], denoted by M4 and whose iterative expression is

$$
\begin{aligned}
& y_{k}=x_{k}-F^{\prime}\left(x_{k}\right)^{-1} F\left(x_{k}\right), \\
& z_{k}=y_{k}-\frac{1}{\beta} F^{\prime}\left(x_{k}\right)^{-1} F\left(y_{k}\right), \\
& x_{k+1}=z_{k}-F^{\prime}\left(x_{k}\right)^{-1}\left((2-1 / \beta-\beta) F\left(y_{k}\right)+\beta F\left(z_{k}\right)\right),
\end{aligned}
$$

where $\beta$ is an arbitrary complex parameter, $\beta \neq 0$. As the authors proved in [5], the corresponding scheme for $\beta=1 / 5$ is the unique element of the family with order of convergence five. In this paper, we will analyze the real dynamical behavior of this family of methods applied to the nonlinear equation $f(x)=0$. We will denote by $G_{f}(x, \beta)$ the fixed point operator associated to the class of methods (1) on a nonlinear real function $f(x)$, whose expression is

$$
G_{f}(x, \beta)=x-\frac{1}{f(x)}\left[f(x)+f(y)(2-\beta)+\beta f\left(y-\frac{1}{\beta} \frac{f(y)}{f^{\prime}(x)}\right)\right]
$$

where $y=x-x-\frac{f(x)}{f^{\prime}(x)}$.

\footnotetext{
This research was supported by Ministerio de Ciencia y Tecnología MTM2011-28636-C02-\{01, 02\}

${ }^{*}$ Corresponding author

Email addresses: alberto.magrenan@gmail.com (Alberto Magreñán), acordero@mat.upv.es (Alicia Cordero), jmguti@unirioja.es (José M. Gutiérrez), jrtorre@mat.upv.es (Juan R. Torregrosa)
} 
The authors in [20] have studied the complex dynamics of the rational function associated to (1), analyzing the conjugacy classes. In it, some complex regions with rich dynamical behavior have been shown providing interesting elements of the family of iterative methods. In this paper, our aim is the real dynamics of this family. Despite what might seem, some numerical results obtained in this paper allows us to conjecture that the real behavior is not included in the complex one. In fact, we will find stable real regions where attracting periodic orbits appear in the complex study, and vice versa.

The dynamical behavior associated to two rational operators (corresponding to a particular iterative method) conjugated by an affine map is qualitatively the same if a Scaling Theorem is satisfied.

Theorem 1. Let $f(x)$ be an analytic function and let $A(x)=a x+b$ with $a \neq 0$ be an affine map. Let $g(x)=(f \circ A)(x)$. Then, $\left(A \circ G_{g} \circ A^{-1}\right)(x)=G_{f}(x)$, that is, $G_{f}$ and $G_{g}$ are affine conjugated by $A$.

Proof: Let us consider firstly

$$
G_{f}(A(x))=T_{f}(A(x))-\frac{\left(2-\frac{1}{\beta}-\beta\right) f\left(N_{f}(A(x))\right)+\beta f\left(T_{f}(A(x))\right)}{f^{\prime}(A(x))},
$$

where $T_{f}$ and $N_{f}$ are the fixed-point operators of the first and second steps, respectively.

As $g(x)=(f \circ A)(x)$, it is clear that $g^{\prime}(x)=a f^{\prime}(A(x)), N_{g}(x)=x-\frac{A(x)-N_{f}(A(x))}{a}$ and also $g\left(N_{g}(x)\right)=$ $f\left(N_{f}(A(x))\right)$. Then,

$$
T_{g}(x)=x-\frac{1}{a f^{\prime}(A(x))}\left[f(A(x))+\frac{1}{\beta} f\left(N_{f}(A(x))\right)\right]
$$

and $g\left(T_{g}(x)\right)=f\left(T_{f}(A(x))\right)$.

Finally,

$$
\begin{aligned}
A\left(G_{g}(x)\right) & =a G_{g}(x)+b \\
& =a\left[T_{g}(x)-\frac{\left(2-\frac{1}{\beta}-\beta\right) g\left(N_{g}(x)\right)+\beta g\left(T_{g}(x)\right)}{g^{\prime}(x)}\right]+b \\
& =G_{f}(A(x))
\end{aligned}
$$

by simply substituting the previous calculations.

The Scaling Theorem allows us to reduce the study of the dynamics of the iteration function $G_{f}$ to the study of specific families of iterations of simpler maps. For example, any real quadratic polynomial $p(x)=c_{2} x^{2}+c_{1} x+c_{0}$ with $c_{2} \neq 0$ (we may assume that $c_{2}=1$ ) can be reduced to $x^{2}+c$, by using an affine map. Then, we are going to analyze the following polynomials that correspond to the three different cases: $p_{-}(x)=x^{2}-1$ a particular case of two different real roots, $p_{+}(x)=x^{2}+1$ (a case with two complex roots) and $p_{0}(x)=x^{2}$, corresponding to multiple roots.

In this paper, we are going to analyze the real dynamics of the set of methods (1) when they are applied to quadratic polynomials, studying the stability of all the real fixed points. The graphic tool used to analyze the stability regions is called the convergence plane and it was introduced by Magreñán in [19].

Now, we are going to recall some dynamical concepts that we use in this work (see [7]). Given a rational function $R: \mathbb{R} \rightarrow \mathbb{R}$, the orbit of a point $z_{0} \in \mathbb{R}$ is defined as:

$$
\left\{z_{0}, R\left(z_{0}\right), R^{2}\left(z_{0}\right), \ldots, R^{n}\left(z_{0}\right), \ldots\right\} .
$$

We analyze the phase plane of the map $R$ by classifying the starting points from the asymptotic behavior of their orbits. A $z_{0} \in \mathbb{R}$ is called a fixed point if $R\left(z_{0}\right)=z_{0}$. A periodic point $z_{0}$ of period $p>1$ is a point such that $R^{p}\left(z_{0}\right)=z_{0}$ and $R^{k}\left(z_{0}\right) \neq z_{0}$, for $k<p$. A pre-periodic point is a point $z_{0}$ that is not periodic but there exists a $k>0$ such that $R^{k}\left(z_{0}\right)$ is periodic. A critical point $z_{0}$ is a point where the derivative of the rational function vanishes, $R^{\prime}\left(z_{0}\right)=0$. Moreover, a fixed point $z_{0}$ is called attractor if $\left|R^{\prime}\left(z_{0}\right)\right|<1$, superattractor if $\left|R^{\prime}\left(z_{0}\right)\right|=0$, repulsor if $\left|R^{\prime}\left(z_{0}\right)\right|>1$ and parabolic if $\left|R^{\prime}\left(z_{0}\right)\right|=1$.

The basin of attraction of an attractor $\alpha$ is defined as:

$$
\mathcal{A}(\alpha)=\left\{z_{0} \in \mathbb{R}: R^{n}\left(z_{0}\right) \rightarrow \alpha, n \rightarrow \infty\right\} .
$$

The Fatou set of the rational function $R, \mathcal{F}(R)$, is the set of points $z \in \mathbb{R}$ whose orbits tend to an attractor (fixed point, periodic orbit or infinity). Its complement in $\mathbb{R}$ is the Julia set, $\mathcal{J}(R)$. That means that the basin of attraction of any fixed point belongs to the Fatou set and the boundaries of these basins of attraction belong to the Julia set. 
When an iterative method is applied to solve a generic polynomial equation $p(z)=0$, its roots are fixed points of the associated rational function $R$. Nevertheless, other fixed points of $R$ different from the roots of $p(z)$ can appear. These fixed points are called strange fixed points. Moreover, it is relevant the knowledge of the free critical points (critical points different from the associated to the roots) : each invariant Fatou component is associated with, at least, one critical point (see, for example [7]).

The rest of the paper is organized as follows: in Section 2 we analyze the fixed and critical points of the operator $G_{f}(x, \beta)$ and the stability of fixed points on quadratic polynomials. The dynamical behavior of the family (1) is analyzed in Section 3, by using the associated convergence plane. We finish the work with some remarks and conclusions.

\section{Study of the fixed and critical points}

As we have stated in the previous section, we will analyze the behavior of the rational function obtained when the family of iterative methods 1 is applied on the polynomials $p_{0}(x), p_{+}(x)$ and $p_{-}(x)$.

In the case of $p_{0}(x), G_{p_{0}}(x, \beta)=\frac{-1+40 \beta}{128 \beta} x$ is a linear map, whose slope $a(\beta)$ has a hyperbolic dependence of $\beta \neq 0$. Thus, its dynamical behavior as a function of $\beta$ its trivial, and it is well-known that, when the origin is stable, it is globally stable; when it is unstable $\left(\frac{-1}{88}<\beta<\frac{1}{168}\right)$ everything not fixed is divergent. For $\beta=-\frac{1}{88}$, when $a(\beta)=1$, all the points are fixed, while for $\beta=\frac{1}{168}$, that is, $a(\beta)=-1$ all the points different from the origin are two-periodic.

The polynomial $p_{+}(x)$ does not have real roots, therefore it can be proved that the rational operator

$$
G_{p_{+}}(x, \beta)=-\frac{1+(3+8 \beta) x^{2}+(3+32 \beta) x^{4}+(1+88 \beta) x^{6}-64 \beta x^{7}}{64 \beta x^{6}}
$$

has three different strange fixed points, depending on $\beta$, but all of them are complex numbers for all values of $\beta$.

Finally, we are going to analyze the dynamics of the operator $G_{f}(x, \beta)$ on the polynomial $p_{-}(x)$. In this case, the rational function associated to the family is

$$
G_{p_{-}}(x, \beta)=\frac{-1+4 x^{2}+8 \beta x^{2}-6 x^{4}-40 \beta x^{4}+4 x^{6}+120 \beta x^{6}-x^{8}+40 \beta x^{8}}{128 \beta x^{7}} .
$$

In addition of the roots of $p_{-}(x), x=1$ and $x=-1$, the fixed points of the operator are, the roots of the polynomial $-1+3 x^{2}+8 \beta x^{2}-3 x^{4}-32 \beta x^{4}+x^{6}+88 \beta x^{6}$. Nevertheless, four of them are always complex so, the only real strange fixed points are:

$$
\begin{aligned}
e x_{1}(\beta) & =-\left[\frac{1}{1+88 \beta}+\frac{32 \beta}{3(1+88 \beta)}-\frac{52 \sqrt[3]{2} \beta}{(1+88 \beta) B_{2}(\beta)}-\frac{272 \sqrt[3]{2} \beta^{2}}{3(1+88 \beta) B_{2}(\beta)}+\frac{2 \sqrt[3]{4} B_{2}(\beta)}{(3(1+88 \beta))}\right]^{\frac{1}{2}}, \\
e x_{2}(\beta) & =-e x_{1}(\beta)
\end{aligned}
$$

where

$$
B_{1}(\beta)=\sqrt{27 \beta^{2}+4720 \beta^{3}+203484 \beta^{4}-242880 \beta^{5}+216832 \beta^{6}}
$$

and

$$
B_{2}(\beta)=\left(-27 \beta+2034 \beta^{2}-2144 \beta^{3}+3 \sqrt{3} B_{1}(\beta)\right)^{1 / 3} .
$$

It is easy to see that $e x_{1}(\beta)$ and $e x_{2}(\beta)$ only take real values for $\beta>0$ or $\beta \in(-1 / 88,0)$. For $\beta=-1 / 88$, the expression of the rational function is

$$
G_{p_{-}}(x, \beta)=\frac{11-43 x^{2}+61 x^{4}-29 x^{6}+16 x^{8}}{16 x^{7}}
$$

and then, the unique real fixed points are $x=1$ and $x=-1$.

In order to analyze the stability of the strange fixed points in the values of the parameter where they are real, we calculate the stability function $S_{i}(x, \beta)=\left|G_{p_{-}}^{\prime}\left(e x_{i}, \beta\right)\right|, i=1,2$. We conclude that $S_{1}(x, \beta)=S_{2}(x, \beta)>1$ for all values of $\beta$. So, the real strange fixed points are always repulsive.

The previous statements can be summarized in the following result.

Lemma 1. The number of real simple strange fixed points of $G_{p_{-}}(x, \beta)$ is

- zero if $\beta \leq-\frac{1}{88}$, 
- two, $\operatorname{ex}_{1}(\beta)$ and $e x_{2}(\beta)$, when $\beta>0$ or $\beta \in(-1 / 88,0)$.

Moreover, for all values of parameter $\beta$, the strange fixed points are repulsive.

Now, in order to determine the critical points, we calculate the first derivative of $G_{p_{-}}(x, \beta)$,

$$
G_{p_{-}}^{\prime}(x, \beta)=\frac{\left(-1+x^{2}\right)^{3}\left(-7+(-1+40 \beta) x^{2}\right)}{128 \beta x^{8}}
$$

From $G_{p_{-}}^{\prime}(x, \beta)$, the free critical points that is, critical points different from the roots, are analyzed in the following result.

Lemma 2. a) If $\beta \leq \frac{1}{40}$ or $\beta=\frac{1}{5}$, then there is no real free critical points.

b) If $\beta>\frac{1}{40}$, the real free critical points are

(i) $\operatorname{cr}_{1}(\beta)=-\frac{\sqrt{7}}{\sqrt{-1+40 \beta}}$,

(ii) $c r_{2}(\beta)=-c r_{1}(\beta)$.

In Figure 1 the behavior and relations of the real strange fixed and free critical points are showed, where $e x_{1}(\beta)$ and $e x_{2}(\beta)$ are presented in red and blue, respectively, while the free critical points have been colored in orange and green. It is known that there is at least one critical point associated with each invariant Fatou component. When an attracting

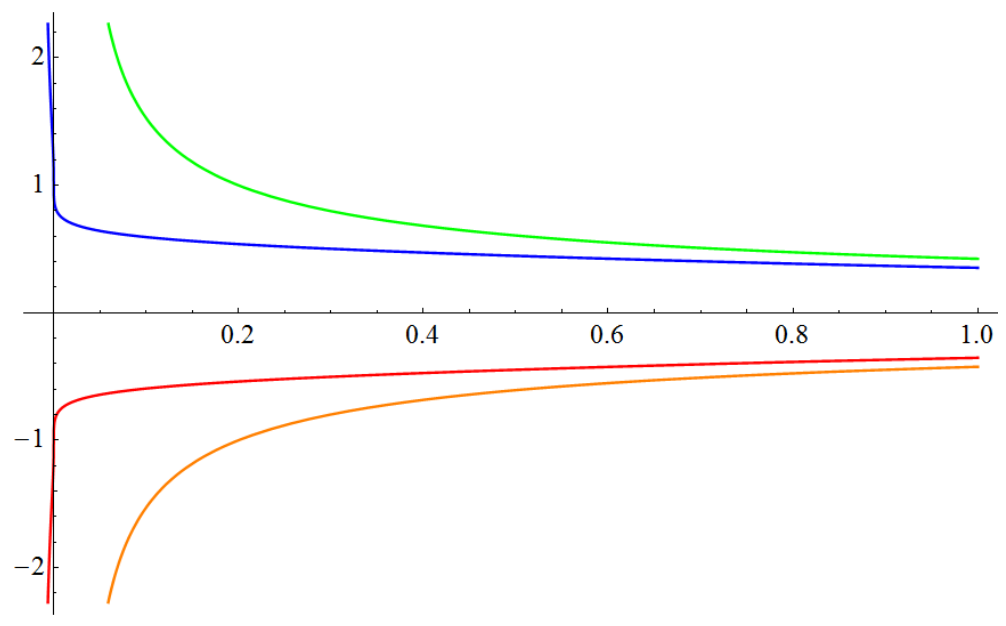

Figure 1: Behavior of the strange fixed and critical points

(but not superattracting) strange fixed points appears, it is important to know if there exist any critical point in its vicinity, because this fact provides that the fixed point has its own basin of attraction.

As it can be inferred from Lemmas 1 and 2, in the rational function associated to the class of iterative methods (1) there are no strange fixed points that are attractive and, consequently, the dynamical planes for particular values of the parameter would have only two basins of convergence. However, as we will see in the following sections, this is not always the case.

In contrast with the real analysis, when a complex dynamical analysis is made (see [20]), there exist some regions where two of the strange fixed points can be attractive, even superattractive. The fixed points are, in all cases, complex numbers.

\section{Convergence Plane for quadratic polynomials}

In Section 2, we have seen that the Scaling Theorem reduces the study of quadratic polynomials to the study of the three following polynomials: $p_{0}(x)=x^{2}, p_{+}(x)=x^{2}+1$ and $p_{-}(x)=x^{2}-1$, which represent respectively polynomials with no real roots, polynomials with one double root and a polynomial with two different real roots. Specifically, in this section we are going to analyze the dynamics of the operator $G_{f}(x, \beta)$ applied to those polynomials.

In the complex plane, the parameter space associated with a free critical point is obtained by associating each point of the parameter plane with a complex value of the parameter and is used to give rise about the dynamics, but in the real 
line we can't use it. Instead of using the parameter spaces we are going to apply the tool called The convergence plane introduced by Magreñán in [19]. This tool gives information about how are the real dynamics for every member of the family and every initial point, so we can find the best choices of the family for each initial point and viceversa. So, it is interesting to find regions of the convergence plane in which the basin of the roots are as much bigger and connected as possible, because these values of the parameter will give us the best members of the family in terms of numerical stability and convergence.

In order to deep in the study of the dynamical behavior of the operator $G_{f}(x, \beta)$, we are going to analyze now the convergence plane associated to it. The key to obtain this convergence plane is to study the orbits of each initial point (horizontal axis) for every member of the family (vertical axis). We paint a duplet $\left(x_{0}, \beta_{0}\right)$, after 1000 iterations and with a tolerance of $10^{-6}$, in cyan if the iteration of the member of the family with $\beta=\beta_{0}$ starting in $z_{0}=x_{0}$ converges to the fixed point 1 , in magenta if it converges to -1 and in yellow if the iteration diverges to $\infty$. Moreover, we paint in red the convergence to any of the strange fixed points, in orange the convergence to 2-cycles, in light green the convergence to 3 -cycles, in dark red to 4-cycles, in dark blue to 5-cycles, in dark green to 6-cycles, dark yellow to 7-cycles, and in white the convergence to 8-cycles. The areas in black correspond to zones of convergence to other cycles.

In order to check the results obtained by the convergence plane we will compare them with the ones from two classical tools: Feigenbaum diagrams and Lyapunov exponents.

Definition 1. Let be $\left\{x_{1}, x_{2}, \ldots, x_{n}, \ldots\right\}$ an orbit. We call Lyapunov exponent

$$
h\left(x_{1}\right)=\lim _{n \rightarrow \infty} \frac{1}{n}\left(\log \left|f^{\prime}\left(x_{1}\right)\right|+\log \left|f^{\prime}\left(x_{2}\right)\right|+\cdots+\log \left|f^{\prime}\left(x_{n}\right)\right|\right) .
$$

Note, that this value is the same for every point of the orbit.

The applicability of the Lyapunov exponents resides on the following result (cf [15] or [19]).

Theorem 2. An orbit $\left\{x_{1}, x_{2}, \ldots, x_{n}, \ldots\right\}$ is chaotic if the two following conditions hold:

- The orbit is not asymptotically periodic.

- $h\left(x_{1}\right)>0$.

So, if the associated Lyapunov exponent to $x_{1}$ is negative, the orbit of $x_{1}$ is attracted by a fixed point, a strange fixed point or by a cycle, but if the exponent is positive the orbit can diverge to infinity or even have a chaotical behavior.

\subsection{Convergence Plane for quadratic polynomials without real roots}

The next case of study corresponds with the case without real roots. That is we are going to analyze the dynamics of the operator $G_{p_{+}}(x, \beta)$.

In Figure 2 we see the convergence plane associated to $G_{p_{+}}(x, \beta)$. It is clear that there is no convergence to any root, but we have found that there exists non-chaotical regions which corresponds to some attracting cycle. In the right of Figure 2 we observe the negative region in which there exist attracting cycles.

Again in order to compare results we see in the Figure 3 are shown the Lyapunov exponents and Feigenbaum diagram associated to $G_{p_{+}}(x, \beta)$. In view of Figure 3 we can conjecture that this polynomial has a very complicated dynamics but it is not chaotical for all values of $\beta$. EXPLICAR FIGURAS 4 Y 5.

\subsection{Convergence Plane for quadratic polynomials with two different real roots}

In this Section we are going to analyze the dynamics of the operator $G_{p_{-}}(x, \beta)$, which constitutes the richest dynamical case.

In Figure 4 we see the convergence plane associated to operator $G_{p_{-}}(x, \beta)$. The first conclusion at the first glance of the convergence plane is that main numerical problems reside in values of $\beta$ close to 0 . So we will focus first our attention in that region. Moreover, we see that it seems that for negative values of $\beta$ the connected basins of attraction are bigger and there exist less changes between one basin to another which reduces the Julia set (as it is defined as the boundary of the basin of attraction). In view of the convergence plane for members of the family $\beta<-\frac{1}{88}$, the iteration of initial points $x_{0}<0$ converge to the root -1 and the iterations of $x_{0}>0$ converge to the root 1 as it can be seen in Figure 5 . This fact does not agree with the behavior in the complex dynamics (see [20]) since the authors found regions in which there exists convergence to different cycles. As a consequence, it seems that the real behavior of the methods is better the the complex behavior. This fact reinforces the idea of real dynamics is not included in complex dynamics. 


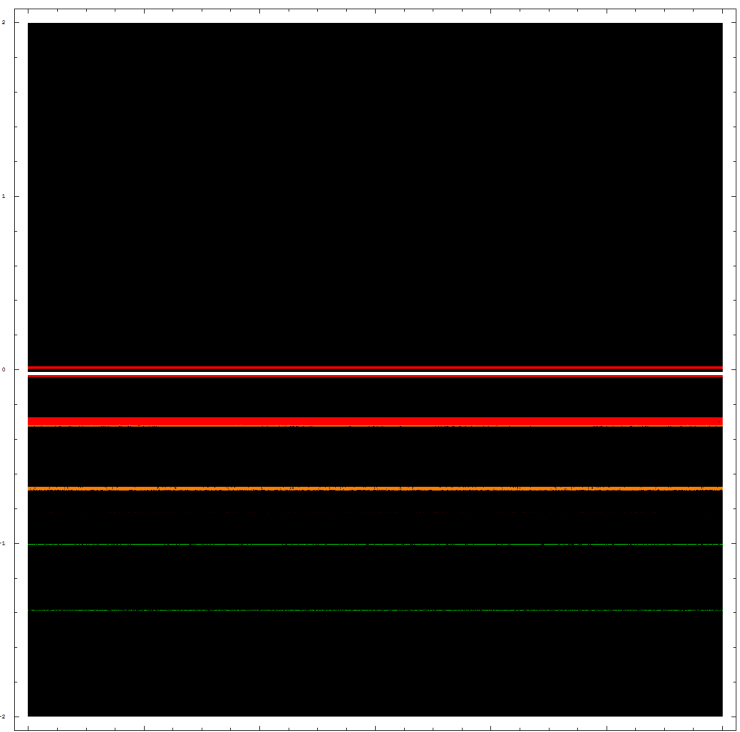

Convergence plane

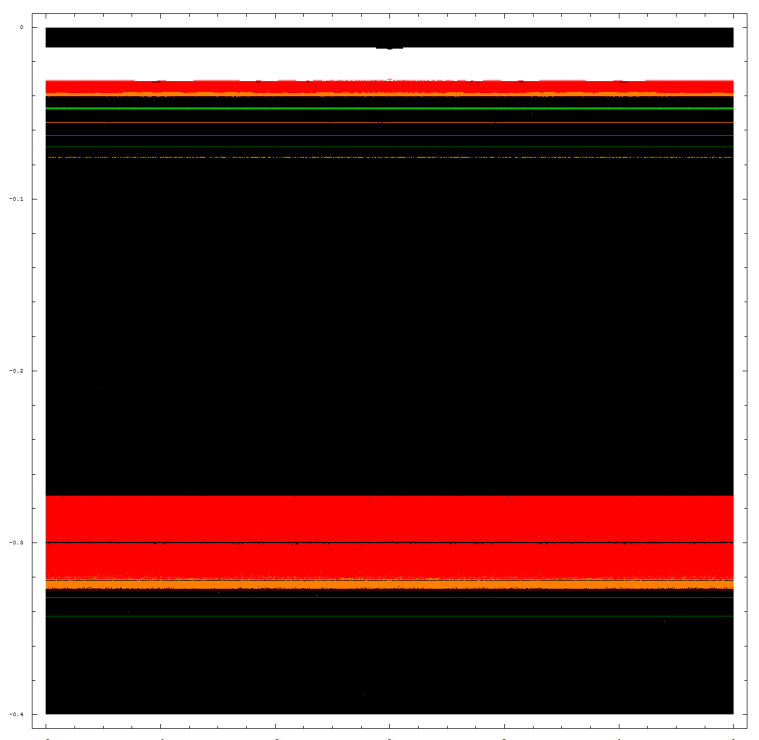

A detail

Figure 2: Convergence plane associated to iteration of the operator $G_{p_{+}}(x, \beta)$

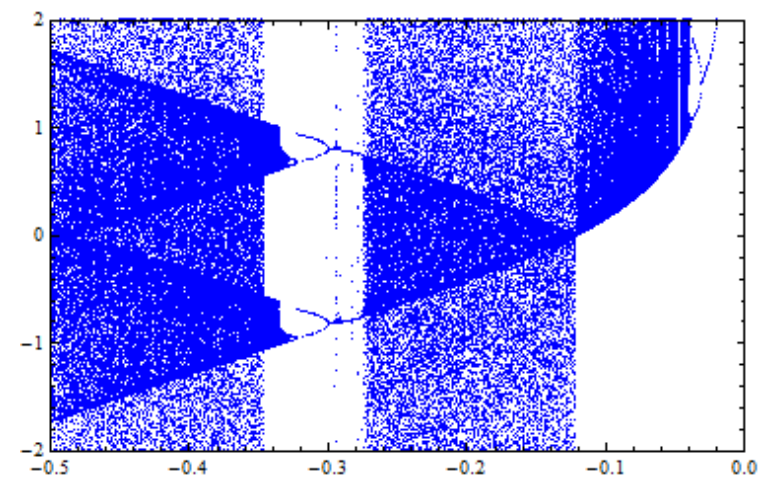

Feigenbaum diagram

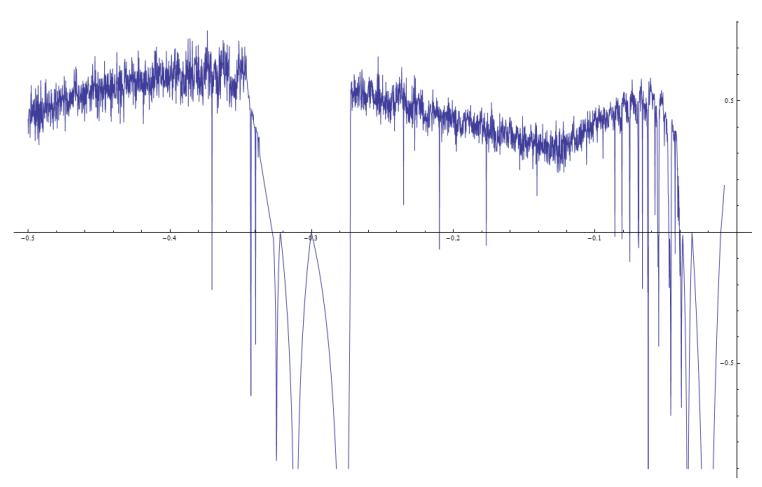

Lyapunov exponents

Figure 3: Feigenbaum diagram and Lyapunov exponents associated to $G_{p_{+}}(x, \beta)$.

Now, we focus our attention in the zone where parameter $\beta$ is close to 0 . In Figure 6 we can see a region of values of $\beta \in(-1 / 88,1 / 164)$ for which there exists divergence to $\infty$ (the yellow region). Those members of the family are not good in terms of convergence.

On the other hand, if we center our attention in the region $\beta \in(1 / 164,0.03)$ we see that there exist some areas in which there seems that the Julia set is a Cantor as can be seen in Figure 7 and there also exists convergence to different cycles as it is shown in Figure 8. As a consequence, every point of the plane which is neither cyan nor magenta is not a good choice of $\beta$ in terms of numerical behavior. Moreover, in Table 1 we show some of the values of $\beta$ for which attractive cycles of different orders appear. Let us note that the period of the orbits is even in all the cases.

Finally, for values of $\beta<-\frac{1}{88}$ or $\beta>0.03$ there is no convergence problems, that is, the iteration of every point converges to one root. This behavior can be seen in Figure 9

A direct conclusion from to the study of Figure 9 is that for values of $\beta<-\frac{1}{88}$ the dynamical behavior seems to be better than for $\beta>0.3$ in terms of stability, since Julia set is more complicated in the last case. For the members of the family such that $\beta<-\frac{1}{88}$ we see that there only exist 2 basins of attraction which correspond with the semiplane that contains the root and so, dynamics is trivial. In the other case, for $\beta>0.3$, we observe that the basins of attraction are not connected and as a consequence the complexity of the Julia set associated is higher. 


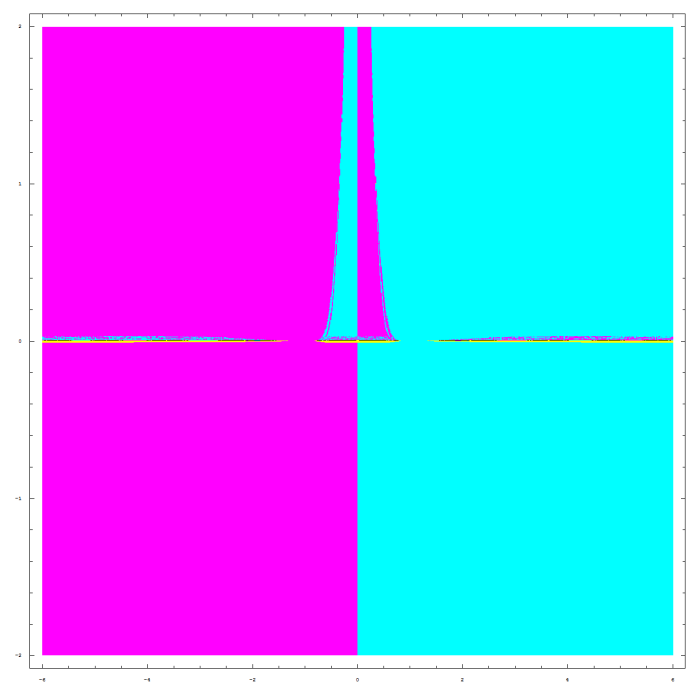

Figure 4: Convergence plane associated to iteration of the operator $G_{p_{-}}(x, \beta)$.

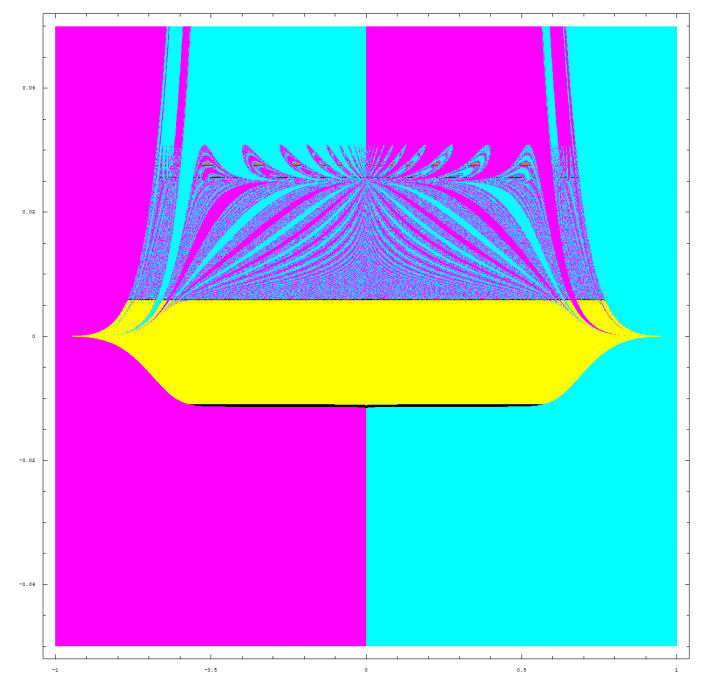

Figure 5: Convergence plane associated to iteration of the operator $G_{p_{-}}(x, \beta)$.

Table 1: Values of $\beta$ and the attractive cycle associated to that value.

\begin{tabular}{|cc|}
\hline$\beta$ & Cycle \\
\hline \hline 0.025650 & $\{-2755.95,-21.8255,-0.271408,2037.03,16.1325,0.260879\}$ \\
0.025700 & $\{0.271359,-2035.85,-17.3296,-0.271359,2035.85,17.3296\}$ \\
0.027690 & $\{-0.49924,10.0582,0.508803,-8.18013,-0.497276,10.4904,0.513692,-7.34945\}$ \\
0.027691 & $\{-0.499238,10.0582,0.508902,-8.16226,-0.497341,10.4753,0.513614,-7.36176\}$ \\
0.027710 & $\{0.511141,-7.76619,-0.499043,10.0924\}$ \\
0.027720 & $\{-0.499656,9.95647,0.510672,-7.84291\}$ \\
\hline
\end{tabular}

\section{Conclusions}

In this paper, we analyzed the real dynamical behavior of the family $M_{4}$ introduced in [5] in terms of convergence and stability, the complex dynamical behavior was studied in [20]. Some numerical experiments allow us to conjecture that real dynamics is not included in complex one, since we have found that in the real line for negative values of $\beta$ there are no cycles although the study of the complex dynamics stated that there exist different cycles. This conclusion would not be possible without the tool developed in [19] which allows to study real dynamics of a uniparametric family for all values of the parameter and all initial points. Moreover, we have showed that values of the parameter close to the origin 


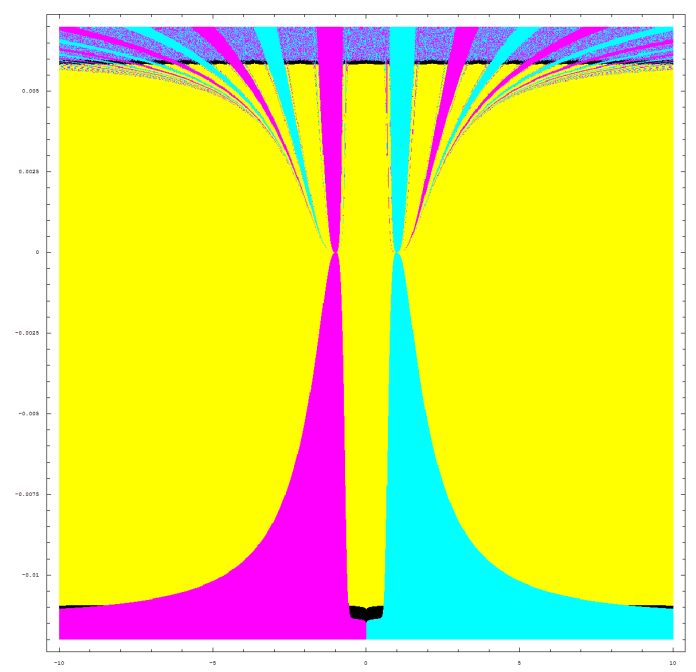

Figure 6: Convergence plane associated to iteration of the operator $G_{p_{-}}(x, \beta)$ in a region close to 0 .

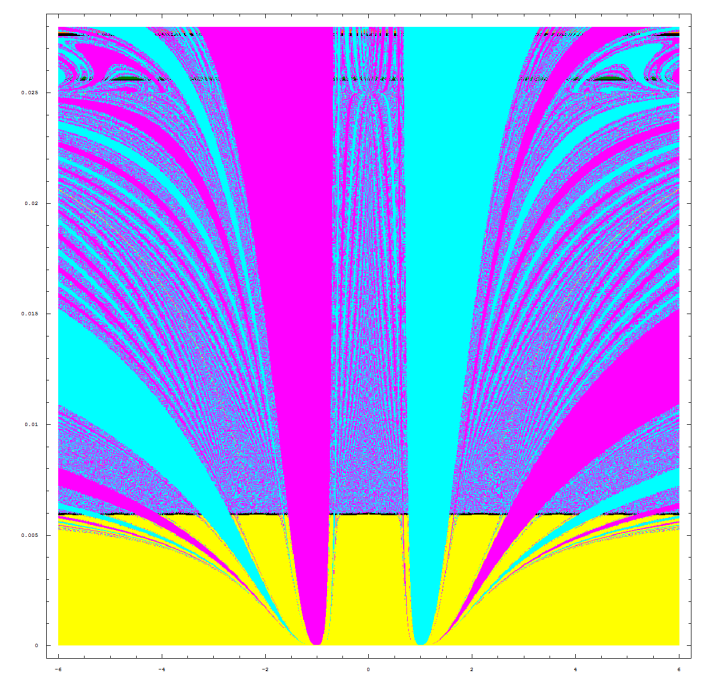

Figure 7: Convergence plane associated to iteration of the operator $G_{p_{-}}(x, \beta)$.

are not appropriate choices due to for that values we have seen that the behavior is complicated as it it shown in [20]. On the other hand we have seen that members of the family $\beta \notin(-1 / 88,0.03)$ the dynamical behavior works properly for both case but, in terms of stability we can state that the best members of the family are those for which $\beta<-1 / 88$.

\section{References}

[1] S. Amat, S. Busquier, S. Plaza, Review of some iterative root-finding methods from a dynamical point of view, Sci. Ser. A: Math. Sci. 10 (2004) 3-35.

[2] S. Amat, S. Busquier, S. Plaza, A construction of attracting periodic orbits for some classical third-order iterative methods, J. Comput. Appl. Math., 189 (2006) 22-33.

[3] S. Amat, S. Busquier, S. Plaza, Chaotic dynamics of a third-order Newton-type method, J. Math. Anal. Appl. 366 (2010) 24-32.

[4] S. Amat, S. Busquier, Á. A. Magreñán, Reducing chaos and bifurcations in Newton-type methods, Abstr. Appl. Anal. Volume 2013 Article ID 726701.

[5] V. Arroyo, A. Cordero, J.R. Torregrosa, Approximation of artificial satellites preliminary orbits: The efficiency Challenge, Math. and Comput. Model. 54 (2011) 1802-1807. 


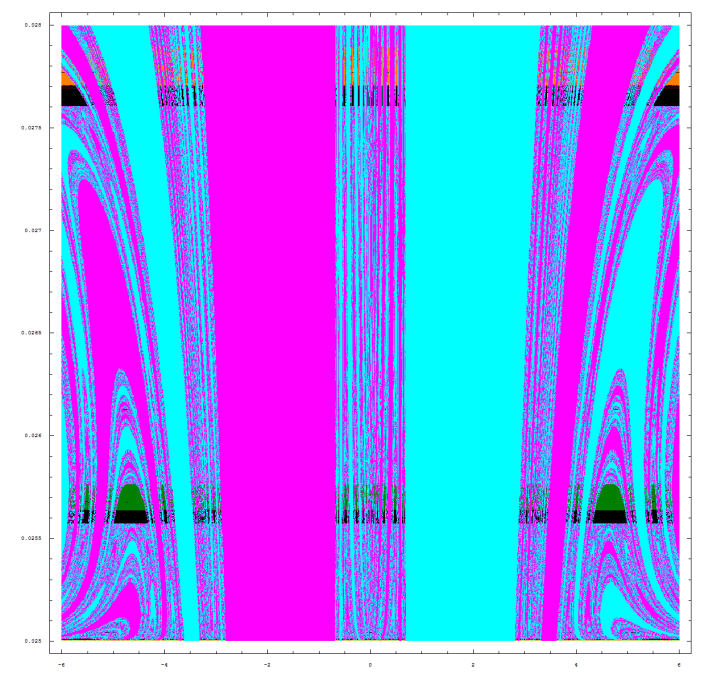

Figure 8: Convergence plane associated to iteration of the operator $G_{p_{-}}(x, \beta)$ in $\beta \in(1 / 164,0.03)$.
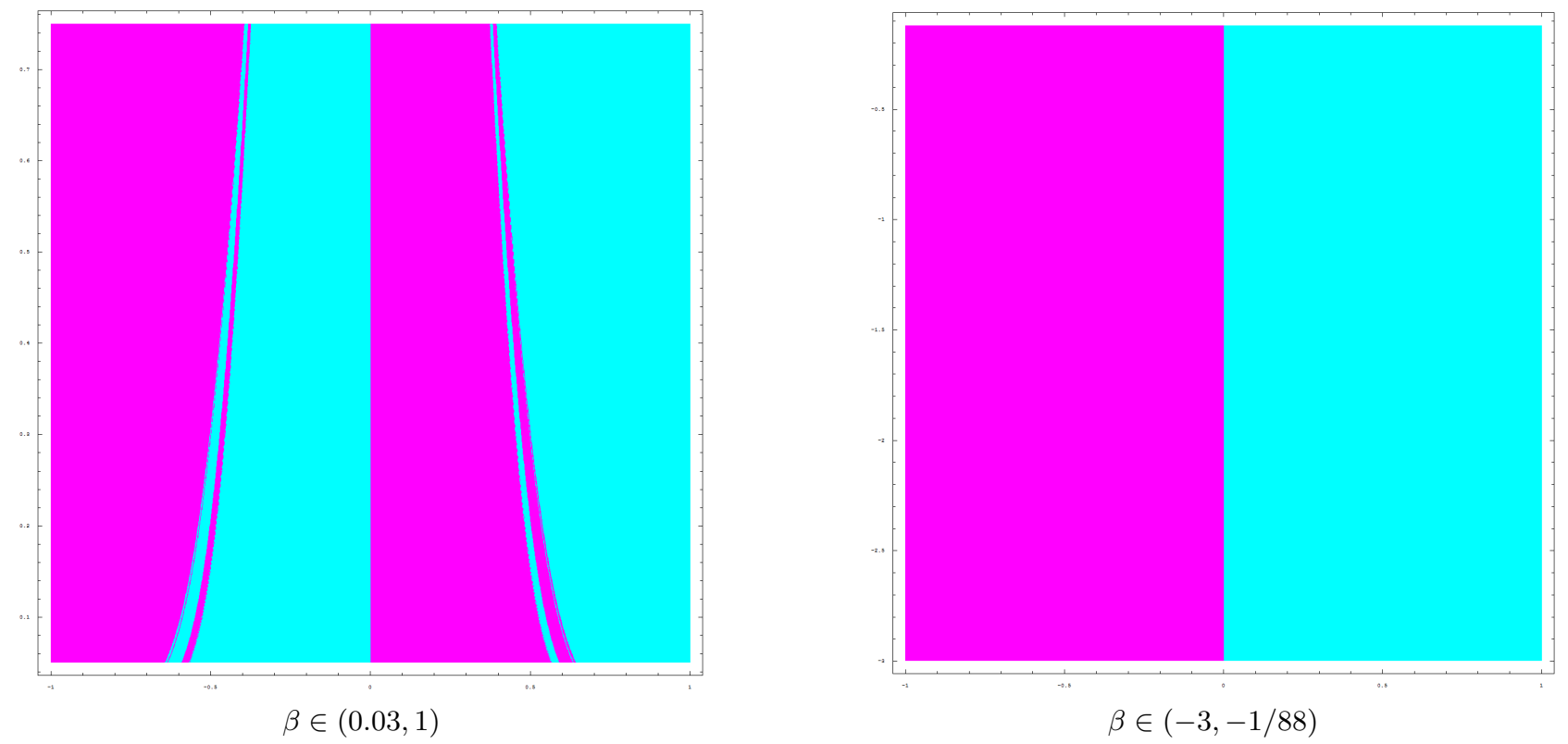

Figure 9: Convergence planes associated to the iteration of the operator $G_{p_{-}}(x, \beta)$.

[6] L. Billings, J.H. Curry, On non-invertible maps of the plane: Eruptions, Chaos 6 (1996) 108-119.

[7] P. Blanchard, Complex Analytic Dynamics on the Riemann Sphere, Bull. of the AMS 11(1) (1984) 85-141.

[8] P. Blanchard, The Dynamics of Newton's Method, Proc. of Symp. in Appl. Math. 49 (1994) 139-154.

[9] F. Chicharro, A. Cordero, J.M. Gutiérrez, J.R. Torregrosa, Complex dynamics of derivative-free methods for nonlinear equations, Appl. Math. Comput. 219 (2013) 7023-7035.

[10] C. Chun, M.Y. Lee, B. Neta, J. Džunić, On optimal fourth-order iterative methods free from second derivative and their dynamics, Appl. Math. Comput. 218 (2012) 6427-6438.

[11] A. Cordero, J. García-Maimó, J.R. Torregrosa, M.P. Vassileva, P. Vindel, Chaos in King's iterative family, Appl. Math. Lett. 26 (2013) 842-848.

[12] A. Cordero, J.R. Torregrosa, P. Vindel, Dynamics of a family of Chebyshev-Halley type methods, Appl. Math. Comput. 219 (2013) 8568-8583. 
[13] R.L. Devaney, The Mandelbrot Set, the Farey Tree and the Fibonacci sequence, Am. Math. Monthly 106(4) (1999) 289-302.

[14] L. Gardini, G. I. Bischi, D. Fournier-Prunaret, Basin boundaries and focal points in a map coming from Bairstow method, Chaos 9(2) (1999) 367-380.

[15] A. Giraldo, M. A. Sastre, Sistemas dinámicos discretos y caos, Fundación general de la Universidad Politécnica de Madrid, Madrid, 2002.

[16] J.M. Gutiérrez, M.A. Hernández, N. Romero, Dynamics of a new family of iterative processes for quadratic polynomials, J. Comput. Appl. Math. 233 (2010) 2688-2695.

[17] J.M. Gutiérrez, S. Plaza, Dinámica del método de newton, Servicio de publicaciones Universidad de La Rioja, 2013. http://dialnet.unirioja.es/servlet/libro?codigo=529750

[18] Á.A. Magreñán, Estudio de la dinámica del método de Newton amortiguado (PhD Thesis), Servicio de Publicaciones, Universidad de La Rioja, 2013. http://dialnet.unirioja.es/servlet/tesis?codigo= 38821

[19] Á.A. Magreñán, A new tool to study real dynamics: The Convergence Plane, arXiv: 1310.3986 [math.NA]

[20] Á.A. Magreñán, A. Cordero, J.M. Gutiérrez, J.R. Torregrosa, Dynamical study of a fourth-order family of iterative methods for solving nonlinear problems, arXiv: 0836444 [math.NA].

[21] B. Neta, M. Scott, C. Chun, Basin attractors for various methods for multiple roots, Appl. Math. Comput. 218 (2012) 5043-5066.

[22] S. Plaza, N. Romero, Attracting cycles for the relaxed Newton's method, J. Comput. Appl. Math. 235 (2011) 32383244.

[23] M. Scott, B. Neta, C. Chun, Basin attractors for various methods, Appl. Math. Comput. 218 (2011) 2584-2599. 